Théologiques

Théologiques

\title{
Les relations entre chrétiens et Juifs depuis 50 ans
} Aperçu historique

\section{Jean Dujardin}

Volume 11, numéro 1-2, automne 2003

Juifs et chrétiens. L’à-venir du dialogue.

URI : https://id.erudit.org/iderudit/009523ar

DOI : https://doi.org/10.7202/009523ar

Aller au sommaire du numéro

\section{Éditeur(s)}

Faculté de théologie de l'Université de Montréal

ISSN

1188-7109 (imprimé)

1492-1413 (numérique)

Découvrir la revue

\section{Citer cet article}

Dujardin, J. (2003). Les relations entre chrétiens et Juifs depuis 50 ans : aperçu historique. Théologiques, 11(1-2), 17-33. https://doi.org/10.7202/009523ar d'utilisation que vous pouvez consulter en ligne.

https://apropos.erudit.org/fr/usagers/politique-dutilisation/ 


\title{
Les relations entre chrétiens et Juifs depuis 50 ans Aperçu historique
}

\author{
Jean Dujardin \\ Prêtre de l'Oratoire \\ Ancien secrétaire du Comité épiscopal français \\ pour les relations avec le judaïsme
}

Faire l'histoire des relations entre chrétiens et Juifs pendant les cinquante dernières années pose diverses questions. Nous n'avons pas la prétention de connaître tous les détails de ce qui s'est passé dans l'ensemble des pays catholiques durant ces années. Notre expérience se limite à ce que nous avons vécu en France. Il n'est pas aisé d'apprécier une mutation si récente, dont la portée n'apparaîtra qu'avec le temps. Il faut nous rappeler d'où nous venons. La pensée la plus courante sur le peuple juif, jusqu'au Concile, acceptait comme une vérité quasi indiscutable le rejet du peuple juif par Dieu et le transfert de sa mission au christianisme. Le judaïsme était devenu incompréhensible, anormal, l'existence juive, coupable, tout espoir d'une restauration sur la terre d'Israël impensable et illusoire. La mission des chrétiens était simple: ils devaient travailler à la conversion du peuple juif. Comment mesurer la portée du changement théologique opéré ?

\section{Les précurseurs}

La périodisation des 50 dernières années se justifie-t-elle pleinement? Pour l'Église catholique, certainement, du point de vue institutionnel en particulier. Personne ne doute de l'importance du signe posé par le pape Jean XXIII lorsqu'il décide de supprimer dans la prière pour les Juifs du Vendredi Saint les mots perfidis et perfidiam en 1959. Ce geste significatif modifie une prière en usage depuis le $\mathrm{VII}^{\mathrm{e}}$ siècle et les transformations successives intervenues depuis jusqu'au texte actuel montrent d'une 
manière éloquente l'évolution théologique accomplie ${ }^{1}$. Mais si l'on se place d'un point de vue chrétien plus large, on ne doit pas oublier les dix points de Seelisberg, résultat de la "Conférence internationale extraordinaire pour combattre l'antisémitisme » qui se tint en Suisse du 30 juillet au 5 août $1947^{2}$.

À l'autre extrémité des 50 années, c'est la demande de pardon déposée au Mur de Jérusalem par le pape Jean-Paul II, le 23 mars 2000, qui fait date. Mais d'un point de vue chrétien à nouveau plus large, il faudrait ajouter un document peu connu mais important, le texte Église et Israël. Contribution des Églises issues de la Réforme en Europe sur les relations entre les chrétiens et les Juifs: "Pour la première fois [en effet], les Églises issues de la Réforme en Europe disposent d'un texte commun adopté à l'unanimité par l'Assemblée des 104 Églises de la communion ecclésiale de Leuenberg réunie à Belfast en juin 20013."

Il n'est donc pas contestable que les 50 dernières années marquent un changement majeur. Mais comme pour tous les changements profonds, il se prépare; c'est pourquoi il est indispensable d'en rappeler les racines lointaines.

Il y a les précurseurs. Chose surprenante, cependant, ce ne sont pas en général des théologiens qui ont ouvert la voie. Ce sont le plus souvent des hommes de lettres, romanciers, philosophes, historiens. Le mouvement s'amorce au XIX ${ }^{e}$ siècle lorsque se développent de nouvelles formes d'antisémitisme. Il prend de l'ampleur avec l'affaire Dreyfus. Les intuitions de ces pionniers qui ne sont pas à l'abri des préjugés antérieurs, posent cependant des jalons parfois fulgurants. Ainsi Anatole LeroyBeaulieu dans son ouvrage Israël chez les nations publié pour la première fois en 1893, un an avant l'affaire Dreyfus, sept ans après la parution de La France juive de Drumont,

1. On lira à ce propos l'étude qui a été faite dans M.-T. НоcH et B. Dupuy, dir., Les Églises devant le judaïsme. Documents officiels 1948-1978, Paris, Cerf, 1980, p. 350-352.

2 Texte dans Hoch et Dupuy, Les Églises devant le judaïsme, p. 19-22.

3. Communion ecclésiale de Leuenberg, Église et Israël. Contribution des Églises issues de la Réforme en Europe sur les relations entre les chrétiens et les Juifs, numéro spécial de Foi et Vie, 101/1 (2002). 
... s'intéresse au Judaïsme comme chrétien; en un temps où la pensée catholique a perdu la notion de sa filiation, et où la controverse et l'apologétique s'attachent plutôt à souligner différences et oppositions, LeroyBeaulieu est de ces chrétiens trop rares qui se souviennent que le christianisme a été enté sur le tronc du judaïsme et qui ne rejetteraient pas la notion de judéo-christianisme dans la meilleure acception du terme [...]. Aucune idée ne revient aussi souvent au cours du livre que le thème de la culpabilité des chrétiens ${ }^{4} \ldots$

Leroy-Beaulieu écrit lui-même ces phrases étonnantes: «À viser le Juif à travers la Bible, c'est risquer de toucher le Christ»; ou encore: "On ne peut atteindre Israël qu'à travers le Christ... Les seuls antisémites logiques sont ceux qui repoussent aussi bien l'Évangile que la $\mathrm{Bible}^{5}$.» Dans la même perspective, un peu plus tard et d'une manière plus passionnée, il faut rappeler les intuitions de Léon Bloy (1846-1917). Certes, dans ce qu'il écrit, il y a des passages inacceptables, mais il y a aussi des phrases fulgurantes comme celle-ci, oh combien célèbre: «L'histoire des Juifs barre l'histoire du genre humain comme une digue barre le fleuve, pour en élever le niveau.» Ou encore: "J'affirme qu'il est aussi téméraire de toucher à la Race juive qu'au Saint Sacrement. » Il répète avec insistance le fait que «Jésus est le juif par excellence», que Marie est «la fleur de la racine juive». Et enfin, plus provoquant: "Sachez que je mange chaque matin un juif qui se nomme Jésus-Christ ${ }^{6}$.»

Parmi ces prophètes, la figure de Charles Péguy — dont Pierre Pierrard écrit: « $[\ldots]$ le seul chrétien notable qui ait regardé ses frères juifs avec un regard clair illuminé de l'intérieur et qui les ait aimés pour euxmêmes $^{7} »-$ mérite une attention spéciale. Péguy intervient dans les tourments de l'affaire Dreyfus, et ses intuitions sont innombrables et lumineuses.

4. R. Rémond, «Préface» [de la réédition], dans A. Leroy-Beaulieu, Israël chez les nations, Paris, Calmann-Lévy, $1983^{2}$ (1893), p. 19, 29.

5. Leroy-Beaulieu, Israël chez les nations, p. $19 \mathrm{~s}$.

6. P. PIERrard, Juifs et catholiques français. D'Édouard Drumont à Jacob Kaplan (1886-1994), Paris, Cerf, 1997, p. 218-220. Pour une étude plus approfondie sur Léon Bloy, on se rapportera à D.R. Goitein, "Léon Bloy et les juifs", dans M. Arveiller et P. Glaudes, dir., Léon Bloy (Cahiers de l'Herne 55), Paris, l'Herne, 1988, p. 280-293.

7. Pierrard, Juifs et catholiques français, p. 222. 
Dans l'entre-deux-guerres et dans le renouveau biblique, des ouvertures apparaissent. On pense aux nombreux travaux du père Joseph Bonsirven depuis son premier livre sur Les Ruines du temple: le judaïsme après Jésus-Christ (1928) jusqu'à Exégèse paulinienne et exégèse rabbinique $(1939)^{8}$. À la même époque, alors que l'antisémitisme s'affiche avec virulence, on n'oubliera pas le combat d'un Oscar de Ferenzy, les écrits et les conférences de Jacques Maritain. Avec lui s'élabore pour la première fois une pensée construite sur le judaïsme. Elle influencera plusieurs des pères du Concile. Dans le combat que Maritain poursuit pendant la guerre à partir des États-Unis, d'autres figures doivent être mentionnées. Ils s'appellent Fessard, Richard, Chaine, de Lubac, de Montcheuil. Ils sont relayés en Suisse par Journet ${ }^{10}$. Ils n'ont certes pas encore l'audience dont ils bénéficieront après la guerre, mais ce sont des hommes d'envergure dont la lucidité et le courage posent les jalons nécessaires et fondent la résistance spirituelle de nombreux chrétiens ${ }^{11}$. Il ne faut pas voir dans leurs écrits une anticipation pleinement élaborée de ce qui adviendra par la suite. Ils demeurent relativement isolés dans une masse chrétienne encore imprégnée d'un enseignement négatif. Mais leurs ouvertures préparent l'avenir ${ }^{12}$.

8. J. Bonsinven, Sur les ruines du temple (le judaïsme après Jésus-Christ) (La vie chrétienne 5), Paris, B. Grasset, 1928; idem, Exégèse rabbinique et exégèse paulinienne (Bibliothèque de théologie historique), Paris, Beauchesne, 1939.

9. Sur l'œuvre de Maritain en ce domaine lire J. Maritain, Le Mystère d'Israël, Paris, Desclée de Brouwer, 1990 (1965); idem, L’impossible antisémitisme, précédé de P. Vidal Naquet, Jacques Maritain et les Juifs, Paris, Desclée de Brouwer, 1994.

10. On lira avec intérêt le point de vue de plusieurs d'entre eux exposé dans H. de Lubac, dir., Israël et la Foi chrétienne, Fribourg, Librairie de l'Université, 1942. Lire aussi Les théologiens lyonnais et la persécution contre les Juifs, Table ronde tenue le 20 janvier 1993 en commémoration de la protestation du cardinal Gerlieren, septembre 1942 (Les Cahiers de l'Institut Catholique de Lyon 25), Lyon, Université catholique de Lyon, 1994.

11. Voir à ce sujet J. Prévotat, "Quatre Jésuites devant le totalitarisme nazi", dans P. Bolle et J. Godel, dir., Spiritualité, théologie et résistance. Yves de Montcheuil, théologie an maquis du Vercors, Colloque de Biviers, Grenoble, Presses Universitaires de Grenoble, 1987, p. 98.

12. Sur ce point, pour un jugement nuancé, voir notre étude: J. Dujardin, L'Église catholique et le peuple juif, Paris, Calmann-Lévy, 2003, p. 54s. 


\section{Premières prises de position des Églises} avant et pendant la guerre: comment les interpréter?

Pour être équitable, on ne saurait oublier dans ce rappel les prises de position de la hiérarchie catholique dans l'entre-deux-guerres. Il y a la condamnation du Saint Office en 1928, la célèbre déclaration de Pie XI aux pèlerins belges en 1938, et un certain nombre de déclarations d'évêques en France et en Allemagne autour des années 30. Toutefois la hiérarchie catholique se tait à partir de 1933 en Allemagne, de 1940 en France. À part la prise de position publique très ferme de l'épiscopat hollandais contre la déportation des Juifs de leur pays le 26 juillet 1942, les évêques des divers pays d'Europe ne reprendront pas la parole. En France, six seulement protestent au cours de la même année 1942 devant des faits patents de persécution. Ce silence pose question et son interprétation est délicate. Il ne signifie pas une complaisance à l'égard de la pensée nazie, impossible à envisager depuis l'encyclique Mit Brennender Sorge (1937). Pourtant, on constate l'absence d'un jugement ferme sur les législations antisémites: lois de Nuremberg, législation antisémite de Vichy et du gouvernement fasciste italien. Dès lors il convient de s'interroger sur la portée exacte des condamnations évoquées plus haut. Elles dénoncent le caractère païen de l'antisémitisme moderne et nazi, mais elles ne remettent pas en cause l'antijudaïsme chrétien dans sa formulation religieuse, pas plus qu'elles ne s'interrogent sur les attitudes que les chrétiens ont adoptées au fil des siècles. On devine cette carence dans la déclaration de Pie XI du 6 septembre 1938. Il dit d'une part avec force: «... l'antisémitisme est inadmissible. Nous sommes spirituellement des sémites ", alors qu'il vient d'affirmer: "Nous reconnaissons à quiconque le droit de se défendre, de prendre les moyens de se protéger contre tout ce qui menace ses intérêts légitimes ${ }^{13}$.» Cette réserve, peut-être compréhensible dans le contexte d'une immigration étrangère intense, comment a-t-elle été reçue dans les consciences chrétiennes, et même dans la hiérarchie? L'épiscopat français, dans ces débats au moment de la promulgation du $1^{\text {er }}$ statut concernant les Juifs en 1940, fera référence aux mesures légales contre les Juifs en vigueur dans les États pontificaux jusqu'en 1870. Dans le projet d'encyclique contre l'antisémitisme, rédigé par les pères jésuites

13. "À propos de l'antisémitisme. Pèlerinage de la Radio catholique belge ", Documentation Catholique, no 885 (5 décembre 1938) p. 1461. 
à la demande de Pie XI, nous retrouvons la même fermeté contre l'antisémitisme nazi et en même temps la persistance des jugements négatifs de la tradition théologique avec les stéréotypes habituels ${ }^{14}$. Faut-il s'en étonner? On trouve sous la plume d'un des rédacteurs du projet, le père Gundlach, cette distinction fort ambiguë entre «... un antisémitisme de type raciste et ethnique incompatible avec l'enseignement de l'Église et un antisémitisme, sinon tout à fait chrétien, du moins acceptable par l'Église, visant à préserver la société chrétienne avec "des moyens moraux et légaux" de l'influence néfaste des Juifs dans la vie chrétienne ${ }^{15}$ ». Les pasteurs pouvaient-ils éclairer les consciences chrétiennes par de tels propos? Ils rendaient possible la collusion inévitable entre l'héritage ancien de l'antijudaïsme et les théories nouvelles. De toute façon, même si on pensait devoir s'opposer à l'antisémitisme dont les conséquences extrêmes n'étaient pas encore perçues, on se méfiait des Juifs, on ne les aimait pas.

Dans l'appréciation de cette période, il faut reconnaître qu'une partie notable de nos frères protestants virent plus clairs que nous. Il faut rappeler le rôle de Karl Barth en Allemagne, son influence sur l'Église confessante. En France, les prises de position mesurées du pasteur Bœgner, et celles exprimées dans ce qu'on appelle les thèses de Pomeyrol ont certainement contribué à soutenir l'action des chrétiens protestants en faveur des Juifs ${ }^{16}$.

Dans un environnement si complexe, les précurseurs ne pouvaient pas être pleinement entendus de la grande masse des chrétiens et c'est pourquoi la charité concrète heureusement active en faveur des Juifs opprimés ne s'est pas pour autant accompagnée d'un changement de regard sur le judaïsme.

14. Voir l'analyse synthétique de l'article du Lexicon für Theologie und Kirche dans DujARdin, L'Église catholique et le peuple juif, p. 498, note 68. L'article intégral est cité dans G. Passelece et B. Suchecky, L'Encyclique cachée de Pie XI: une occasion manquée de l'Église face à l'antisémitisme (L'Espace de l'histoire), Paris, Découverte, 1995, p. 94-95.

15. G. Gundlach, «Antisemitismus», Lexikon für Theologie und Kirche, Fribourg, Herder, 1930, p. 504-505.

16. Voir à ce sujet P. Bolle, "Les thèses de Pomeyrol ", dans Bolle et Godel, dir., Spiritualité, théologie et résistance, p. 172-174. 


\section{Jean XXIII et le Concile Vatican II}

La prise de conscience se fit après la guerre devant l'horreur de l'extermination. C'est à Seelisberg et dans une perspective analogue que Jean XXIII et le Concile inaugurèrent l'étape décisive. Mais comment le Concile a-t-il abouti à la déclaration Nostra Ætate, $\mathbb{S} 4$ ? Il faut faire droit, pour répondre à cette question, au travail remarquable de Jules Isaac. Rien ne préparait cet historien laïc a priori à un tel travail, sinon sa présence aux côtés de son ami et compagnon Charles Péguy, au moment de l'affaire Dreyfus. C'est la guerre, la déportation et la mort de sa femme et de sa fille, qui le déterminèrent à écrire ce qui deviendra en 1948 Jésus et Israël. Interrogation profonde et douloureuse sur les racines religieuses de l'antisémitisme, ce livre commence par une citation révélatrice de N. Berdiaeff: «Nul ne doute que par rapport au peuple d'Israël, les chrétiens portent un lourd péché. [...] L'antisémitisme à base religieuse [est] le plus sérieux et le seul qui mérite d'être étudié ${ }^{17}$. " Très convaincu de cette dimension essentielle du problème, Jules Isaac écrit le 7 janvier 1946: «Tant que les Églises et leurs fidèles n'auront pas pris conscience de ces responsabilités initiales chrétiennes, l'anti-judaïsme gardera sa virulence ${ }^{18}$. Jules Isaac résume sa pensée dans une formule restée célèbre: les chrétiens sont responsables de l'«enseignement du mépris ». Les propositions de son livre inspirèrent Seelisberg. Mais il ne s'en tint pas là. Après l'annonce de la convocation d'un concile par Jean XXIII, il fut convaincu qu'il y avait là une occasion d'agir. Il prépara un mémorandum pour le Pape et fut reçu en audience le 13 juin 1960. À la fin de l'entretien, le Pape lui dit: «Vous avez droit à plus que de l'espoir.»

Se pose alors cette question: d'où provenait l'intérêt du Pape? Rien dans son parcours intellectuel et spirituel ne l'y avait préparé. Certes, on le sait, son regard sur les autres, personnes et groupes humains, a toujours été empreint d'une profonde bonté. Il n'y avait pas d'a priori antijuif chez lui. Sa bonté à leur égard s'exprima pendant son séjour à Istanbul. Il nota au cours d'une retraite en 1940, après avoir reçu des

17. J. IsaAc, Jésus et Israël, Paris, Albin Michel, 1948, p. 1 (la citation de N. BerdiaefF est extraite de son article "Le Problème juif", Christianisme social, 52 (1939) p. 303 et 310).

18. Documents intégralement cités dans Dujardin, L'Église catholique et le peuple juif, p. 420-439. 
nouvelles de la persécution en Pologne: «le sentiment patriotique qui est légitime et peut être saint, peut dégénérer en nationalisme [...]. Le monde est intoxiqué par un nationalisme malsain, fondé sur la race et le sang, en contradiction avec l'Évangile. » Apprenant en 1943 le naufrage du Struma, qui emportait 769 réfugiés juifs, il écrit: "Nous sommes confrontés à l'un des plus grands mystères de l'histoire de l'humanité. Pauvres enfants d'Israël. J'entends tous les jours leurs gémissements autour de moi. Puisse le divin Sauveur leur porter la lumière. » Les mots «mystère" et "Israël» disent quelque chose de sa perception du caractère exceptionnel de l'événement. Il ne remet pas totalement en cause l'enseignement traditionnel. Il écrit même: "La reconstruction du royaume de Judée et d'Israël n'est qu'une utopie ${ }^{19}$. " Cependant, ici comme dans tout le déroulement de sa vie, de par sa formation d'historien, il a le sens du mouvement, sa pensée n'est pas figée. Cela se traduit par la volonté constante d'être attentif à l'action de l'Esprit saint, par la volonté de discerner les signes des temps ${ }^{20}$. On peut penser que cela le prédisposait à prêter une attention particulière aux remarques de Jules Isaac. Et de même qu'il introduit dans la préparation du Concile une donnée nouvelle avec la nomination du cardinal Bea à la tête du Secrétariat pour l'unité des chrétiens, il confie à ce dernier la responsabilité de préparer un document sur les Juifs.

L'élaboration en fut lente. Nostra Ætate connut sept versions avant d'être définitivement adoptée ${ }^{21}$. Les difficultés se situaient à des niveaux divers. Opposition théologico-politique des Églises orientales d'abord. Réelle et forte jusqu'au bout. Ces Églises craignaient que le document apparaisse comme une justification a posteriori par le Concile de l'existence et de la politique de l'État d'Israël. L'attitude des États arabes et des manifestations opportunes alimentait leurs craintes. Mais il faut voir aussi dans leur opposition le lourd héritage antijuif des Pères d'Orient.

19. On trouvera une explication sur ce problème entre autres dans l'ouvrage du Grand Rabbin Jacob Kaplan, Justice pour la foi juive, Paris, Cerf, $1995^{2}$ (1977), p. 167 205. Le Grand Rabbin a été un acteur personnel dans cette affaire.

20. Lire à ce propos la biographie de P. Hebblethwaite, Jean XXIII, le pape du Concile, Paris, Bayard, 2000 (1988, anglais 1984).

21. Voir A.-M. Henry, Les relations de l'Église avec les religions non chrétiennes (Unam Sanctam 61), Paris, Cerf, 1966. 
Leur poids théologique posait un problème d'envergure. Pouvait-on faire fi de leur pensée? Obstacle qui rejoignait l'opposition de la minorité conservatrice du Concile, qui voyait dans la Déclaration, comme elle la verra dans celle sur la «Liberté religieuse ", une infidélité à la tradition. Nostra Ætate, $\mathbb{S} 4$, contrairement aux autres documents conciliaires, ne se réfère à aucun texte patristique, aucun document conciliaire, aucune déclaration pontificale. Elle s'appuie sur la Bible, le Nouveau Testament et surtout sur l'épître aux Romains de Paul. Le Concile s'est trouvé en quelque sorte devant un vide et un trop-plein. Depuis Actes 15, aucune réflexion théologique du Magistère suprême - conciles œcuméniques ou papes - ne s'était interrogée sur la nature de la relation qui demeurait entre le peuple juif et l'Église depuis la séparation de fait. Mais dans le même temps, le vide avait été comblé par des mesures disciplinaires discriminatoires innombrables prises contre les Juifs, y compris par des conciles œcuméniques à Bâle, à Latran IV. La pensée commune s'exprimait dans la théologie de la substitution. Comment sortir d'une telle impasse?

C’est la réflexion conciliaire sur l'Église (avec la remise en valeur, dès le premier chapitre de Lumen Gentium, de la notion de «Mystère »); le rappel dans Lumen Gentium, $\mathbb{S} 16$, à propos d'Israël, que les dons et l'appel de Dieu sont «sans repentance» (Rm 11,28-29); la clarification apportée par Dei Verbum sur les rapports entre Écriture et Tradition (notamment au $\mathbb{S}$ 9) qui permirent, du point de vue théologique, au Concile de surmonter la difficulté. Ces concepts de «Mystère» et de «Tradition» (mémoire) dans une sens renouvelé, sont inscrits dans la première phrase du $\mathbb{S} 4$ de Nostra Ætate: "Scrutant le Mystère de l'Église, le Concile se souvient du lien qui relie spirituellement le peuple du Nouveau Testament avec la lignée d'Abraham.» Le rapport entre l'Église et le peuple juif appartient au dessein de salut du Père (le Mystère), il s'établit par un acte de mémoire, lequel nous est confié par le Fils, cette relation «spirituelle» est œuvre de l'Esprit. Plus tard, le pape Paul VI, dans une lettre à $\mathrm{M}^{\mathrm{gr}}$ Lefebvre, exprimera d'une manière profonde la conception de la Tradition que le Concile a remise en évidence. Il lui écrit :

La Tradition n'est pas une donnée figée ou morte, un fait en quelque sorte statique qui bloquerait, à un moment déterminé de l'histoire, la vie de cet organisme actif qu'est l'Église [...]. Il revient au Pape et aux Conciles de porter un jugement pour discerner dans les traditions de l'Église ce à quoi 
il n'est pas possible de renoncer sans infidélité au Seigneur et à l'Esprit Saint [...] ce qui peut et doit être mis à jour pour faciliter la prière et la mission de l'Église à travers les temps ${ }^{22} \ldots$ [nous soulignons]

\section{La Shoah: l'événement-choc}

La question demeure cependant: comment cette lecture est-elle devenue possible à ce moment précis? Dans Nostra Ætate, il n'est fait aucune référence à ce qu'on appelle maintenant la Shoah. Le père Congar aurait même dit: "Auschwitz n'est pas une catégorie de la théologie.» Certes, tout nous donne à penser que l'événement a joué un rôle de dévoilement et permis une nouvelle écoute de la Parole, car il était très présent dans la mémoire des pères conciliaires qui avaient vécu la guerre. Il y était fait référence dans les dix points de Seelisberg. Il demeure cependant difficile d'en mesurer l'impact. À ce moment-là, cet impact est sans doute plus «ressenti " comme la nécessité d'une réparation que «réfléchi» dans sa portée. Aujourd'hui, après une étude plus approfondie, il nous semble se situer à trois niveaux. La Shoah révèle que le peuple juif fut l'objet de cette extermination à cause de la permanence de sa vocation de témoin et non pas seulement du fait de son appartenance à «une race de soushommes». Nous le savons par les textes: c'est à cause de sa fidélité obstinée à la transcendance du Dieu unique, et de son rôle de témoin de la transcendance de la Loi qu'on a voulu le faire disparaître ${ }^{23}$. Le peuple juif est le témoin gênant. Il est, selon l'expression de Hitler, «l'antihomme ", c'est-à-dire le rejet de sa conception de l'homme. Et par là, la vocation juive réapparaît dans son caractère positif. La conséquence inévitable en est un positionnement différent de l’Église. (1) Elle ne renonce en rien à ce qu'elle est, mais elle se souvient de la place du peuple juif dans le dessein de Dieu, d'où une attitude plus humble. (2) On ne peut plus parler de manière simpliste de l'infidélité d'Israël et de la fidélité des chrétiens, comme si des chrétiens ne s'étaient pas trouvés du côté des

22. Paul VI, «Lettre de Paul VI à Mrr Lefebvre », Documentation Catholique, $\mathrm{n}^{\circ} 1710$ (19 décembre 1976) p. 1058.

23. Nous ne pouvons pas développer dans cet article ce qui nous a conduit à cette conclusion. Nous renvoyons pour ce faire à notre analyse: DuJARdin, "L'Église et le peuple juif, un autre regard", dans L'Église catholique et le peuple juif, p. 23-64. 
persécuteurs, comme le disait Jules Isaac, par infidélité à l'Évangile. (3) Enfin, au cœur de l'événement réapparaît le lien oublié qui nous relie comme chrétiens à la racine sur laquelle nous sommes greffés. Expression significative, Hitler ne voulait pas seulement éliminer le peuple juif, mais «l'éradiquer de l'histoire» et ainsi vider de sa substance le christianisme lui-même. Nouvelle manifestation du marcionisme, Hitler suggère en 1934 de remplacer l'Ancien Testament par Mein Kampf dans la Bible de l'Église nationale, ce qui suscita la réaction de l'Église confessante.

Ce qui a été élaboré à Vatican II apparaît de ce fait d'une portée inouie. Lors d'un colloque sur les racines de l'antijudaïsme en milieu chrétien, en préparation au Jubilé de l'an 2000, le père J.M. Garrigues a pu résumer le déplacement opéré par la formule: «le Mystère du peuple juif dans le plan de salut, négation théologique de sa permanence et redressement magistériel».

\section{Après Vatican II et dans la mouvance du Concile}

À partir de là, un autre rapport commence à s'établir entre chrétiens et Juifs. Il fut d'abord le fait d'initiatives «privées ». Jules Isaac, avec l'appui d'amis chrétiens, créa l'association interconfessionnelle chrétienne et juive qu'on appelle l'Amitié judéo-chrétienne de France, avec son équivalent sur le plan international ${ }^{24}$. Les Pères de Sion publièrent pendant plusieurs années une remarquable revue d'éveil et de réflexion: les Cahiers Sioniens. Les Sœurs de Sion créèrent à Rome, puis à Paris et dans plusieurs pays où elles étaient implantées, des centres d'études appelés SIDIC (Service international de documentation judéo-chrétienne). Puis, peu à peu, des évêques des épiscopats nationaux s'exprimèrent ${ }^{25}$. Parmi tous les textes, on doit faire mention de celui qui fut publié sous la responsabilité de la Conférence épiscopale française le 16 avril 1973. Le titre, Orien-

24. Voir à ce sujet A. KAsPI, Jules Isaac, historien, acteur du rapprochement judéochrétien, Paris, Plon, 2002. Lire aussi tous les numéros de la revue SENS (revue de l'Amitié judéo-chrétienne de France) consacrée à ce sujet ainsi que la publication faite par la même revue de la correspondance entre J. Isaac et les principaux acteurs de ce travail.

25. On en trouvera les principaux documents dans le livre de Églises devant le judaïsme. 
tations pastorales $d u$ Comité épiscopal français pour les relations avec le judaïsme ${ }^{26}$, est trop modeste et dit peu de choses sur la richesse d'un contenu qui va au-delà de simples indications pastorales, puisqu'il ose aborder, pour la première fois, la question du retour du peuple juif sur sa Terre et celle de la signification théologique de la permanence d'Israël.

L'année suivante, le Saint-Siège créait la Commission du Saint-Siège pour les relations religieuses avec le judaïsme. Cette commission était chargée de l'application pastorale de Nostra Ætate, $\mathbb{S} 4$. Elle a publié un premier document d'application en 1975 , un second en $1985^{27}$.

\section{Les difficultés du dialogue}

En 1971 se met en place un Comité international de liaison entre les représentants officiels de l'Église catholique et l'International Jewish Committee for Interreligious Consultations. La première réunion se tint à Paris du 14 au 16 décembre 1971, d'autres suivirent dans divers pays européens ou sur d'autres continents à un rythme assez régulier. Un vrai dialogue a-t-il commencé? Oui, en ce sens que pour la première fois dans l'histoire, Juifs et catholiques se rencontrent officiellement et sur un pied d'égalité pour se parler sur des sujets choisis en commun, dans une atmosphère d'attention mutuelle, sans perspective de prosélytisme. Mais pas encore, dans le sens où il ne s'agit pas d'un dialogue théologique à proprement parler, car les chrétiens et les Juifs n'ont pas les mêmes attentes. Dans une conférence récente, le cardinal Cassidy rappelle l'objection du grand rabbin Toaff: "Une discussion théologique n'est pas possible car c'est précisément là que nos chemins divergent et où un accord reste impossible; un accord signifierait, soit que nous renoncerions $\left[\right.$ sic] à notre position, soit que l’Église renonce à la sienne ${ }^{28}$. " La perspective d'un dialogue proprement théologique est-elle définitivement

26. Conférence épiscopale françaIse, Orientations pastorales du Comité épiscopal pour les relations avec le judaïsme (16 avril 1973); texte publié dans Documentation Catholique, no 1631 (6 mai 1973) p. 419-422.

27. Le texte de 1975 se trouve dans l'ouvrage de Dupuy et $\mathrm{Hoch}$, Les Églises devant le judaïsme, p. 353-362. Celui de 1985 a été publié dans Documentation Catholique, no 1900 (21 juillet 1985) p. 733-738.

28. E. Toaff et A. Elkann, Essere Ebreo, Milan, Bompiani, 1994, p. 57. Cité dans E. Cassidy, «L'avenir des relations ", Documentation Catholique, no 2160 (18 mai 1997) p. 495. 
écartée? C'est peu probable, mais elle demandera du temps, des clarifications de part et d'autre sur sa portée, un climat de confiance qui permet d'en reconnaître l'intérêt et la possibilité. Le dialogue théologique s'esquisse d'ailleurs dans les échanges individuels ou au sein de groupes informels, notamment à la faveur d'une lecture partagée des Écritures ${ }^{29}$.

Mais il y faudra du temps, car il ne peut pas faire abstraction de la mémoire qui habite les interlocuteurs. On ne dialogue pas comme si le passé allait s'effacer d'un coup et laisser la place à une écoute réciproque totalement neuve. À travers le dialogue, ce sont aussi des mémoires qui se rencontrent. Elles ont chacune leurs caractéristiques. Comme chrétiens, nous n'ignorons pas l'histoire de l'antijudaïsme, mais parce que nous n'en avons pas souffert, nous ne la connaissons que de façon superficielle et nous mesurons mal les séquelles profondes qu'elle a imprimées dans la conscience juive. Nous croyons vite que le changement de notre attitude en permet l'oubli. Or, il n'en est pas ainsi. Le passé ne s'efface pas, il doit être assumé pour faire place à une attitude nouvelle. L'avonsnous d'ailleurs parfaitement assumé, n'a-t-il laissé aucune trace dans nos propres consciences, trace qu'on voit réapparaître devant certaines réactions juives? Le plus difficile à changer, ce sont les mentalités. Les nôtres sont certes en voie de changement, mais nous serions téméraires de croire que le travail est achevé. Quant à la mémoire juive, une mémoire blessée, marquée par les persécutions et la précarité de l'existence juive dans les différents lieux où les événements ont conduit ces membres, elle demeure inquiète. Elle craint la renaissance des anciens jugements ou comportements chrétiens.

\section{Affronter des événements qui nous troublent}

C'est pourquoi des obstacles demeurent. Plusieurs événements des dernières années nous en ont rappelé l'importance. Il y eut, au lendemain de la Deuxième Guerre mondiale, le contentieux au sujet des conversions que l'affaire Finaly ${ }^{30}$ fit renaître. Résolue non sans difficultés, elle laissa sub-

29. Un exemple passionnant de cette approche se trouve dans C. Chalier et M. FaessLER, Judaïsme et christianisme. L'écoute en partage, Paris, Cerf, 2001.

30. Il s'agit d'enfants qui furent confiés à une chrétienne pendant la guerre. Après le conflit, elle s'empressa de les faire baptiser et elle tenta de les soustraire aux membres de la famille survivants. Voir à ce sujet J. Kaplan, L'affaire Finaly, Paris, Cerf, 1993. 
sister le doute et la méfiance. D'autres événements ont ravivé le doute quant à la profondeur du changement survenu dans le monde chrétien. Il faut rappeler la perspective d'une béatification d'Isabelle la Catholique en 1992, ainsi que l'affaire du Carmel d'Auschwitz, perçue comme une tentative de christianisation de la Shoah. Les péripéties malheureuses autour de la publication de la Bible des communautés chrétiennes ${ }^{31}$ justifient aussi l'incertitude: la traduction et les notes, reproduction adaptée en France de l'édition latino-américaine, comportaient ici ou là des clichés antijuifs et, plus profondément, une insuffisante reconnaissance de la valeur propre de l'Ancien Testament dans la ligne du Concile. Dès lors, sa publication conduisait les Juifs à se poser la question suivante: où trouve-t-on le véritable enseignement de l'Église? Est-ce dans les textes officiels bienveillants qui nous sont adressés ou plutôt dans la permanence d'un enseignement à connotation antijuive? L'incompréhension juive toute récente devant la béatification conjointe de Jean XXIII et de Pie IX constitue un autre fait révélateur. On peut sans peine imaginer que d'autres événements ou décisions raviveront la mémoire juive et qu'un changement, vieux seulement de quelques décennies et dont on sait qu'il n'a pas encore pénétré l'ensemble du monde chrétien, demandera du temps avant d'être pleinement assimilé et reconnu.

Il y a enfin l'obstacle créé par les souffrances qui résultent du conflit entre Israéliens, Palestiniens et Arabes. Certes, depuis 1993, l’Église catholique a reconnu l'État d'Israël. Mais elle est restée longtemps silencieuse et perplexe quant à la signification du retour du peuple juif sur sa terre. À part le texte des évêques français, elle n'a rien dit jusqu'en 1985. Ses réticences tiennent évidemment à la situation politique et aux injustices qui en sont le fruit, mais aussi à la vision religieuse de la condition juive dispersée qu'il fallait remettre en question. Le doute, dès lors, demeure chez beaucoup de Juifs sur l'acceptation profonde de leur rassemblement. Ils gardent en mémoire le silence de Paul VI lors de son pèlerinage en Terre sainte en 1964. Et ils n'ignorent pas que devant les événements, beaucoup de chrétiens n'arrivent pas à établir une distinction nette entre la signification religieuse que ce rassemblement peut revêtir pour les Juifs, sa réalisation dans le contexte actuel par la médiation historique d'un État, les choix et les positions politiques discutables

31. La Bible des communautés chrétiennes, Paris, Médiaspaul, 1994. 
des organes du pouvoir. Beaucoup de Juifs se demandent si les chrétiens acceptent vraiment leur présence sur cette terre, s'ils ne s'accommoderaient pas mieux de leur disparition. Inquiétude aggravée par le sentiment que tout ce qui se passe entre eux et les Palestiniens est la faute par excellence des "Juifs». Ainsi sont-ils englobés dans une généralisation meurtrière comme s'ils étaient tous coupables en quelque endroit où ils se trouvent, comme s'ils approuvaient sans débat et sans contestation les choix de leurs dirigeants.

\section{Le rôle exceptionnel du pape Jean-Paul II}

L'ampleur du changement, dont nous savons en même temps la lente progression dans le monde chrétien, on doit pour une part essentielle l'attribuer au rôle exceptionnel du pape Jean-Paul II. Non seulement il a pleinement assumé l'orientation conciliaire, mais il s'y réfère constamment, il en développe les prémisses dans ses multiples interventions (plus de 150). Il saisit chaque occasion de rencontrer ou d'accueillir des représentants du judaïsme pour encourager le dialogue. Il s'est exprimé avec clarté sur des points incertains, comme la permanence de l'alliance conclue avec Moïse. Il a parlé, dès 1980, de l'existence d'Israël et œuvré avec fermeté pour sa reconnaissance, la situant de façon significative dans l'évolution plus large des relations judéo-chrétiennes. Il a eu le sens des gestes qui touchent le cœur. En 1986, il se rendait à la synagogue de Rome et parlait des «Juifs» comme des «frères aînés ». Premier voyage de cette sorte accompli par un pape dans l'histoire depuis l'apôtre Pierre, il inaugurait en fait de manière discrète une démarche de repentance à laquelle il va donner toute sa signification à l'approche du Jubilé et dont le couronnement se trouve dans le dépôt de sa demande de pardon dans les interstices du Mur occidental, lors de sa visite à Jérusalem, en 2000. Il invite ainsi par sa parole et par son attitude, l'ensemble des épiscopats et les chrétiens à accomplir la même démarche. Il conviait déjà à cette démarche l'Église universelle par le document de la Commission du Saint-Siège, Nous nous souvenons: une réflexion sur la Shoah de mars $1998^{32}$. Ce document n'a pas pleinement satisfait les Juifs et nombre de chrétiens sur plusieurs points. Mais la démarche est là.

32. Commission du SAint-Siège pour les relations AVEC le Judä̈sme, Nous nous souvenons: une réflexion sur la Shoah, texte publié dans Documentation Catholique, $\mathrm{n}^{\circ} 2179$ (5 avril 1998) p. 336-340. 
Il faut reconnaître que ce Pape a mesuré plus que quiconque l'importance d'une "purification de la mémoire ", selon sa propre expression.

\section{Conclusion}

L'histoire des relations entre Juifs et chrétiens a connu depuis 50 ans des développements inimaginables. Mais le renouvellement n'en est qu'à ses débuts. La purification de la mémoire n'est pas achevée et, d'une certaine manière, elle ne s'achèvera jamais pleinement dans la mesure où la rencontre de l'Autre nous appelle à une conversion permanente. La prolongation douloureuse et tragique du conflit israélo-palestinien risque de l'empoisonner pendant longtemps. Le nouvel enseignement de l'Église ne touche qu'un petit nombre de chrétiens et la prise en compte de l'existence juive dans sa positivité n'interpelle pas encore profondément la réflexion théologique. Cependant le Pape la souhaite. Dans plusieurs discours, Jean-Paul II parle de l'Église dans le dessein de Dieu, de l'Incarnation de Jésus comme homme juif. Elle est présente dans le document récent de la Commission Biblique Pontificale, Le peuple juif et ses Saintes Écritures dans la Bible chrétienne ${ }^{33}$. Il faut se rappeler la pensée traditionnelle: les Juifs ont perdu l'usage de leurs Écritures, ils ne savent plus les lire. L'intitulé du document est significatif. Quant au cheminement juif par rapport au dialogue, il me semble qu'il appartient à des Juifs de le décrire et d'en mesurer la portée. Ce que nous en savons nous donne à penser que le dialogue interpelle fortement plusieurs d'entre eux, même s'il faut regarder leur cheminement autrement que le nôtre pour faire droit à leur différence d'attente.

33. Pontificia Commissio Biblica, Le peuple juif et ses Saintes Écritures dans la Bible chrétienne, Città del Vaticano, Libreria Editrice Vaticana, 2001. Voir les articles de M. GiRARD et J. GRUNEWALD dans ce numéro. 


\title{
RÉSUMÉ
}

Comment évaluer la portée du changement théologique opéré par l'Église catholique dans ses rapports avec les Juifs et le judaïsme? D'abord par un effort de mémoire, car cette mutation inouie est récente et fragile; nous n'en saisissons pas encore l'impact à long terme et cependant nous avons déjà de la difficulté à prendre la mesure du chemin parcouru: précurseurs du dialogue; dénonciation résolue de l'antisémitisme pourtant encore teinté d'antijudaïsme, dès avant la guerre; choc de la Shoah et Concile Vatican II.

\begin{abstract}
How can we evaluate the scope of the theological shift in the Catholic Church in its relationship with the Jews and Judaism? Firstly, we must clearly remember that this incredible change is recent and fragile. We still cannot foresee its long term impact while we already have a hard time to acknowledge all that bas been done since the precursors in the dialogue; the denunciation of anti-Semitism, before the war, although still tinged with an anti-Jewish stance; the Shoah and its schock; and Vatican II.
\end{abstract}

